\title{
The association between cerebral developmental venous anomaly and concomitant cavernous malformation: an observational study using magnetic resonance imaging
}

\author{
Guolu Meng, Chuanfeng Bai, Tengfei Yu, Zhen Wu*, Xing Liu, Junting Zhang and Jizong zhao
}

\begin{abstract}
Background: Some studies reported that cerebral developmental venous anomaly (DVA) is often concurrent with cavernous malformation (CM). But there is lack of statistical evidence and study of bulk cases. The factors associated with concurrency are still unknown. The purpose of this study was to determine the prevalence of concomitant DVA and CM using observational data on Chinese patients and analyze the factors associated with the concurrency.

Methods: The records of all cranial magnetic resonance imaging (MRI) performed between January 2001 and December 2012 in Beijing Tiantan Hospital were reviewed retrospectively. The DVA and CM cases were selected according to imaging reports that met diagnostic criteria. Statistical analysis was performed using the Pearson chi-square statistic for binary variables and multivariable logistic regression analysis for predictors associated with the concurrent $\mathrm{CM}$.

Results: We reviewed a total of 165,230 cranial MR images performed during the previous 12 year period, and identified 1,839 cases that met DVA radiographic criteria. There were 205 patients who presented concomitant CM among the 1,839 DVAs. The CM prevalence in DVA cases (11.1\%) was significantly higher than that in the non-DVA cases $(2.3 \%)(P<0.01)$. In the multivariate analysis, we found that DVAs with three or more medullary veins in the same MRI section (adjusted $\mathrm{OR}=2.37,95 \% \mathrm{Cl}$ : 1.73-3.24), infratentorial DVAs (adjusted $\mathrm{OR}=1.71,95 \% \mathrm{Cl}$ : 1.26-2.33) and multiple DVAs (adjusted OR $=2.08,95 \% \mathrm{Cl}$ : 1.04-4.16) have a higher likelihood of being concomitant with CM.

Conclusions: CM are prone to coexisting with DVA. There is a higher chance of concurrent CM with DVA when the DVA has three or more medullary veins in the same MRI scanning section, when the DVA is infratentorial, and when there are multiple DVAs. When diagnosing DVA cases, physicians should be alerted to the possibility of concurrent $\mathrm{CM}$.
\end{abstract}

Keywords: Developmental venous anomaly (DVA), Cavernous malformation (CM), Magnetic resonance imaging (MRI)

\section{Background}

Cerebral vascular malformation (CVM) is a class of important central nervous system diseases. According to Russell and Rubinstein's standard, there are four major types of vascular malformations of the central nervous system: developmental venous anomaly (DVA), cavernous malformation (CM), arteriovenous malformation, and capillary telangiectasia [1]. DVA and CM are the

\footnotetext{
* Correspondence: wuzhen2013mail@sian.com

Department of Neurosurgery, Beijing Tiantan Hospital, Capital Medical University, 6 Tiantan Xili, Chongwen District, Beijing 100050, People's Republic of China
}

two most common diseases among CVM. DVA makes up $42-63 \%$ of all CVM [2]. CM accounts for $5-13 \%$ of all CVM [3].

DVA is a congenital abnormality of venous drainage [4] composed of radially arranged venous complexes converging into a centrally located venous trunk, which drains the normal brain parenchyma [5]. DVAs have a benign clinical course [6], and the reported incidence is $0.05-3 \%$ based on enhanced imaging and autopsy in the general population [6-10]. CM is a vascular lesion that lacks the features of arteries or veins $[11,12]$. CMs occur 
in about $0.4 \%$ of autopsies. The pathogenesis of CM includes pathological factors as well as genetic mutation [13].

Most DVAs and CMs are asymptomatic and are discovered incidentally through neuroimaging. MRI is an effective imaging tool for detecting DVA and CM $[7,14]$ because both DVA and CM have respective characteristic imaging [11]. Other methods such as digital subtraction angiography can be used for diagnosis, but with the wide application of MRI, and noninvasive features, MRI diagnosis is often a preferable alternative. For the purposes of identifying both DVA and CM, MRI is also better suited since most CMs cannot be diagnosed using angiography.

Past studies found that DVA is often concurrent with CM. CM is reported in the literature to have an association with DVA at a rate of $2 \%-33 \%$ [4,12,15-18]. The presence of CM with or without DVA will influence treatment [19]. There are several documented reasons for the concurrency. There is increased systemic or local venous pressure in the DVA [20]. Increased venous pressure may lead to recurrent petechial congestional hemorrhage $[21,22]$, or may produce ischemia which stimulates the growth of new vessels [23]. These new vessels are fragile and susceptible to bleeding, and repeated hemorrhaging may subsequently form a CM $[7,21]$.

In this study, we aimed to determine the prevalence of concurrent DVA and CM using observational data on Chinese patients and to analyze the factors associated with concurrency.

\section{Methods}

\section{Study population and data collection}

From January 1, 2001 to December 31, 2012, a total of 165,230 patients received MRI screening at Beijing Tiantan Hospital. Data were collected from the patients' MRI registration system and MRI reports. For patients who underwent multiple MRI screenings over the study period, only the most recent screening results were included in this analysis. Of these patients, 1,839 patients were diagnosed with DVA.

According to the standard procedures of Beijing Tiantan Hospital, all MRI images were read by two radiologists, including at least one senior-level radiologist. The final diagnosis was approved by both radiologists. In rare cases when the radiologists' diagnosis was inconclusive, the researcher examined the original MRI images and assigned a classification to the case.

\section{MRI screening}

During the study period,the following MRI machines used were: GE Signa 1.5 T, GE Signa 3.0 T, Toshiba Visa1.5 T, and Siemens Magnetom Trio 3.0 T superconducting magnetic resonance imager. The contrast agent was Gadopentetate dimeglumine (Gd-DTPA).

\section{Diagnostic criteria using MRI}

DVA diagnostic criteria included presence of lesions in the white matter, typical stellate or linear vascular lesions converging into a collecting vein and draining into the dural, sinus, or deep veins, and an umbrella or caput medusa-like appearance especially on an enhanced image [7]. If three or more medullary veins were visible simultaneously in at least one section of MRI, and presented in "spoke wheel" or caput medusae configurations [24], we classified the DVA in a " $\geq 3$ medullary veins group". When there were fewer than three medullary veins in any single section of MRI, we classified the DVA in a " $<3$ medullary veins group".

CM diagnostic criteria included presence of lesions with reticulated mixed signal blood-containing locules with the classic heterogeneous "popcorn" appearance on both T1 and T2-weighted images, a rim of haemosiderin in the surrounding brain parenchyma; and minimal or no enhancement on the T1 image [7,9]. Their appearance on a MRI will depend on the degree of the hemorrhage, with T2-weighted images being the most sensitive sequence.

Hemorrhages were not classified as CMs if the hemorrhage lesions were only acute or subacute hematomas dominated by intracellular methaemoglobin, and therefore, appeared with a homogeneous signal on MRI images; and if there were only tiny, punctate foci of hypo intensity on both T1 and T2-weighted sequences, with no heterogeneous signal. On imaging, when CM diagnosis was made, other causes of a single hemorrhagic lesion, such as arteriovenous malformation, bland intraparenchymal hemorrhage, hemorrhagic infection, and neoplasm had to be excluded [18].

\section{Statistical analyses}

We compared different groups using the Pearson chisquare statistic for categorical variables. We also performed multivariable logistic regression analysis to find the factors associated with concurrent CM. Independent variables included age, gender, location of DVA, largest number of medullary veins $(\geq 3$ or $<3)$ in the same MRI section, and quantity of DVAs. For the age variable, age groups were formed by 20 year intervals. For the location variable, patients were divided into two groups, supratentorial and infratentorial. All confidence intervals reported were 95\%, and all p-values were two-sided. P-values less than 0.05 were considered statistically significant. All statistical analyses were performed using SAS software (Version 9.1.3, SAS Institute Inc., Cary, NC, USA).

\section{Ethics}

The study was approved by the Committee on Human Research at Beijing Tiantan Hospital.

\section{Results}

In total, 165,230 patients received MRI screening at Beijing Tiantan Hospital from January 1, 2001 to December 31, 
2012. Of these patients, 1,839 (1.11\%) were diagnosed with DVA and 3,856 (2.33\%) were diagnosed with CM. Among the 1,839 DVA cases,a subgroup of 205 (11.15\%) cases presented with concomitant CM. In the 163,391 non-DVA cases, there were 3,651 cases of CM, and the prevalence was $2.23 \%$. The CM prevalence in DVA cases $(11.15 \%)$ was significantly higher than in the non-DVA cases $(\mathrm{P}<0.01)$.

Among the 1,839 DVA cases, 940 were male (51.11\%) and 899 were female. The average age was 40.40 years (range: $0.25-87$ ) with a standard deviation of 16.01 years. We detected 1,782 (96.90\%) cases with a single DVA, and $57(3.10 \%)$ cases with multiple DVA. Supratentorial and infratentorial DVA cases numbered 1,319 (71.72\%) and $520(28.28 \%)$ respectively. We found 388 (21.10\%) DVA cases with three or more medullary veins visible simultaneously in at least one section of MRI, and 1,451 (78.90\%) DVA cases with fewer than three medullary veins in any single MRI section.

Besides 205 concurrent CMs, there were 174 (9.46\%) cases of concomitant hemorrhage or hematoma with DVAs, but the hemorrhage did not meet our diagnostic criteria for CM in MRI, and thus were not classified as CM.

Among the 205 DVA associated with CM cases,169 (82.44\%) had CMs adjacent to DVAs and located at the distal radicles of DVAs. There were 188 (91.71\%) cases with a single CM lesion, and 17 cases with multiple CMs.

In the multivariate analysis, concomitant CM was associated with three variables. Concomitant CM was almost twice as likely to occur when DVA was in an infratentorial location (adjusted OR $=1.71$, 95\% CI: 1.26 2.33, $\mathrm{P}=0.00$ ). Additionally, $\mathrm{CM}$ was 2.37 times more likely to occur with DVA if there were three or more medullary veins visible in one MRI section (95\% CI: 1.73-3.24, $\mathrm{P}=0.00$ ). Though multiple DVAs were not significantly associated with concomitant $\mathrm{CM}$ in the univariate analysis $(\mathrm{P}=0.051)$, the likelihood of concomitant $\mathrm{CM}$ was 2.08 times more (95\% CI: 1.04-4.16, P = 0.038) holding all other variables constant. Finally, though gender was significantly associated with concomitant CM in the univariate analysis, there was no association in the multivariate analysis (Tables 1 and 2).

\section{Discussion}

Previous studies indicate that DVA and CM are the two most common central nervous system diseases among

\begin{tabular}{|c|c|c|c|c|}
\hline DVA type & $\mathrm{CM}$ & Non- CM & Total & CM prevalence \\
\hline DVA & 205 & 1634 & 1839 & $11.15 \%$ \\
\hline Non-DVA & 3651 & 159740 & 163391 & $2.23 \%$ \\
\hline Total & 3856 & 161374 & 165230 & $2.33 \%$ \\
\hline
\end{tabular}

$X^{2}=617.75, P<0.01$. vascular malformations, and are frequently found coexisting [24]. However, most of these studies were based on small numbers of cases outside of China. The prevalence and imaging features of coexisting DVA and CM in the Chinese population needs to be clarified. Using MRI detection, our study indicates that morbidity of CM in DVA cases is significantly higher than in the non-DVA population. We also found a higher likelihood of concomitant CM when the DVA had three or more medullary veins in the same MRI section, when the DVA was infratentorial, and when multiple DVAs were present.

We found a $1.11 \%$ prevalence of DVA and an even higher prevalence of CM at 2.33\%. In the literature, DVAs are the most common intracranial vascular malformation. There are two possible explanations for this discrepancy. First, our study sample is not representative of the general population. Beijing Tiantan Hospital is one of the largest neurosurgery centers in China, and many patients are referred to this hospital for CM diagnosis and treatment. In comparison, more DVAs are asymptomatic than CMs. Second, all of our patients were from China. It is possible that $\mathrm{CM}$ is the most common intracranial vascular malformation among Chinese people. Among the 1,839 patients with DVA, 205 also had CM. The prevalence of concurrent $C M$ in DVA cases was $11.15 \%$, which is significantly higher than CM non-DVA cases $(2.23 \%, \mathrm{P}<0.01)$. The coexistence of DVA and CM has been recognized by some researchers $[11,17,25,26]$. In DVA cases, the most common coexisting vascular anomaly was CM [24]. CM are reported in the literature to have an association with DVA at a rate of $2 \%-33 \%[4,12,15-18]$. In our study the rate was $11.15 \%$ (205/1839). In most studies, once a hemorrhage or hematoma was detected in DVA cases, the diagnosis of CM would be made $[2,4,21,27]$. In our study, there were also 174 cases of concomitant hemorrhage or hematoma with DVA. However, we did not diagnose these as CM because the hemorrhage did not meet our diagnostic criteria for MRI-detected CM. If we classified patients who had any hemorrhage as CM cases, the concurrent rate would be higher, reaching $20.61 \%$.

The close relationship between CM and DVA may suggest that formation of CM is caused by DVA. Elevated venous pressure in DVA often leads to tiny recurrent hemorrhages [21,22]. Self-limited recurrent hemorrhages [28] may subsequently form a CM [7,21]. Among the 205 coexisting CM cases in DVAs, 169 cases (82.44\%) had CMs adjacent to the DVAs and located at the distal radicles of DVAs. This phenomenon indicates a close relationship between CM and DVA. Thus, follow-up of DVA cases with hemorrhage is warranted because repeated hemorrhage may likely lead to CM formation (Figures 1 and 2).

Our study found a higher likelihood of concurrent CM in DVAs with three or more medullary veins occurring in one MRI section, in infratentorial DVAs, and when 
Table 2 Clinical and imaging predictors of concomitant CM among DVA cases

\begin{tabular}{|c|c|c|c|c|c|c|c|c|}
\hline \multirow{2}{*}{$\begin{array}{l}\text { Characteristics } \\
\text { Overall }\end{array}$} & \multirow{2}{*}{$\begin{array}{l}\text { Total } N \\
N=1839\end{array}$} & \multirow{2}{*}{$\begin{array}{l}\text { CM N (\%) } \\
\mathrm{N}=\mathbf{2 0 5}\end{array}$} & \multirow{2}{*}{$\begin{array}{l}\text { Non CM N (\%) } \\
\mathrm{N}=1634\end{array}$} & \multirow[t]{2}{*}{ P-value } & \multirow[t]{2}{*}{ Unadjusted $\mathrm{OR}^{\dagger}\left(95 \% \mathrm{Cl}^{\S}\right)$} & \multirow[t]{2}{*}{$P$ value } & \multirow[t]{2}{*}{ Adjusted $\mathrm{OR}^{\mp}\left(95 \% \mathrm{Cl}^{\S}\right)$} & \multirow[t]{2}{*}{ P-value } \\
\hline & & & & & & & & \\
\hline Age & . & & & & & & & \\
\hline \multirow[t]{2}{*}{ Median (IQR), years } & $41.5(29-52)$ & $39(27-49)$ & $42(29-52)$ & 0.0242 & & & & \\
\hline & & & & 0.2299 & & & & \\
\hline$<20$ & 210 & $25(11.9 \%)$ & 185(88.1\%) & & 1.0 & . & 1.0 & . \\
\hline 20- 39 & 617 & $80(13 \%)$ & $537(87 \%)$ & & $1.10(0.68-1.78)$ & 0.6899 & $1.06(0.65-1.72)$ & 0.2037 \\
\hline $40-59$ & 801 & $82(10.2 \%)$ & 719(89.8\%) & & $0.84(0.52-1.36)$ & 0.4848 & $0.85(0.53-1.39)$ & 0.6832 \\
\hline$>=60$ & 211 & $18(8.5 \%)$ & 193(91.5\%) & & $0.69(0.36-1.31)$ & 0.2555 & $0.72(0.38-1.38)$ & 0.2760 \\
\hline Gender & . & & & 0.0241 & & & & \\
\hline Female & 899 & $85(9.5 \%)$ & $814(90.5 \%)$ & & 1.0 & . & 1.0 & . \\
\hline Male & 940 & $120(12.8 \%)$ & $820(87.2 \%)$ & & $1.40(1.04-1.88)$ & 0.0246 & $1.30(0.97-1.76)$ & 0.0839 \\
\hline DVA Location & & & & $<.0001$ & & & & \\
\hline Supratentorial & 1319 & $121(9.2 \%)$ & 1198(90.8\%) & & 1.0 & & 1.0 & \\
\hline Infratentorial & 520 & $84(16.2 \%)$ & $436(83.8 \%)$ & & $1.91(1.41-2.57)$ & 0.0000 & $1.71(1.26-2.33)$ & 0.0006 \\
\hline DVA imaging & . & & & $<.0001$ & & . & & \\
\hline Medullary veins $\geq 3$ & 1451 & $128(8.8 \%)$ & 1323(91.2\%) & & 1.0 & . & 1.0 & . \\
\hline Medullary veins<3 & 388 & $77(19.8 \%)$ & $311(80.2 \%)$ & & $2.56(1.88-3.48)$ & 0.0000 & $2.37(1.73-3.24)$ & 0.0000 \\
\hline Quantity of DVAs & & & & 0.0470 & & & & \\
\hline Single & 1782 & 194(10.9\%) & 1588(89.1\%) & & 1.0 & & 1.0 & \\
\hline Multiple & 57 & $11(19.3 \%)$ & $46(80.7 \%)$ & & $1.96(1.00-3.84)$ & 0.0510 & $2.08(1.04-4.16)$ & 0.0384 \\
\hline
\end{tabular}

OR: odds ratio, $\mathrm{Cl}$ : confidence interval, IQR: inter-quartile range.

†Unadjusted odds ratios and $p$-values generated via univariate analysis.

${ }^{\ddagger}$ Adjusted odds ratios and $p$-values resulted from multivariate analysis using a logistic regression model. All variables in univariate analysis were included in the multivariable model.

${ }^{\S} \mathrm{All} \mathrm{Cl}$ presented are $95 \% \mathrm{Cl}$ and all p-values presented are two-sided.

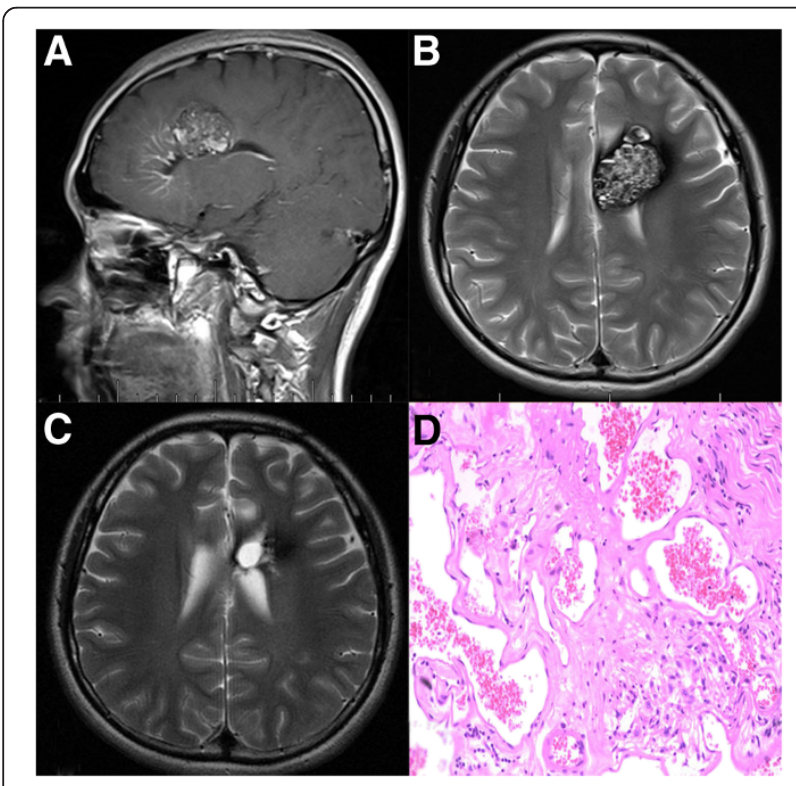

Figure 1 Supratentorial DVA and Concomitant CM. A: Enhanced MRI image showing the DVA and the CM which locates at the distal radicles of the DVA. B: CM shows classic heterogeneous "popcorn" appearance on T2-weighted image. C: Post-operation image showing that the CM disappeared. D: Pathological picture of the CM showing the sinusoidal vascular channels. multiple DVAs were present. The presence of several medullary veins may lead to increased venous pressure and a greater chance of bleeding. While it is difficult to measure the pressure of DVA, CM pressure has been detected during surgery and reported to be markedly higher than cortical venous pressure [29].

When a patient has a DVA that is infratentorial and has three or more medullary veins occurring in one MRI section, there is a $16 \%$ chance of concomitant CM, which is significantly higher than that of supratentorial

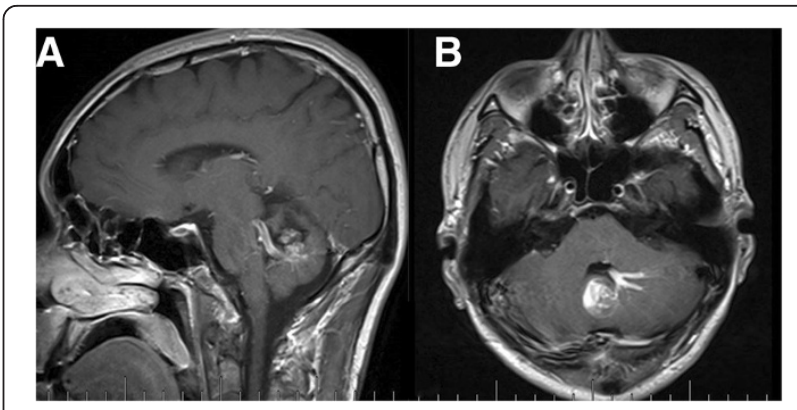

Figure 2 Subtentorial DVA and Concomitant CM. A and B. Enhanced MRI images showing irregular "caput medusae" in the left cerebellar hemisphere and pons, and the CM locates at the distal radicles of the DVA. 
DVA. Studies suggest that DVAs in the posterior fossa are more likely to hemorrhage than their supratentorial counterparts [30].

Multiple DVAs were highly associated with concurrent CMs. No other study has reported this finding. Due to a low proportion of multiple DVAs, this may be a random event. However there is a possibility that multiple DVAs foster a greater opportunity for the development of CM lesions.

The age at presentation of CM is usually between 40 to 60 years [28]. In the literature, the coexistence of CM and DVA is more common in adults than children [31]. We found no statistical significance between the concurrent rates of CM in our four age groups. Moreover, our analysis did not show that gender was related to concomitant CM.

MRI is an extremely sensitive approach for detecting CM [7,14]. Radiologists have strongly recommended diagnosing CM based on characteristic MRI features, thus avoiding additional invasive procedures like digital subtraction angiography and surgical biopsy. The most common appearance of CM is known as a "popcorn" lesion, which involves a nucleus with a heterogeneous signal in T1- and T2-weighted images surrounded by a complete hemosiderin ring with lower signal intensity in T2-weighted sequences [3]. The appearance of CM varies based on the degree of the hemorrhage. CMs have a propensity to grow over time [28]. The typical presence of CM coexisting with DVA may originate from repeated small hemorrhages [17,28,31-33].

Most CMs are solitary lesions, whereas $10-30 \%$ are multiple form [12,14,28,34]. Most cases with a single lesion are sporadic form. This difference in the imaging features of familial and sporadic CM suggests that the two have different developmental mechanisms [12]. Familial cases show more multiplicity and often have a dominant pattern of inheritance due to a gene mutation localizable in the CCM1, CCM2, or CCM3 gene loci $[6,35,36]$. Familial CMs have different pathogenetic mechanisms than sporadic CMs [25]. Among the 205 concurrent CM cases, 188 (91.71\%) cases had a single CM lesion, so it may be inferred that most of our cases are sporadic CMs rather than genetic familial cases. Sporadic CM is highly associated with DVA, because DVA is involved in the formation and development of the sporadic CM.

\section{Limitations}

The results of this study should be interpreted with the following limitations. First, this was a cross-sectional observational study, and data in the patients' registration system and MRI reports were limited. However, the sample size was quite large. Thus, these results may be generalizable to the involving patients. Second, we only used the images from the MRI scan to diagnose cases. Clinical and angiographical data were not included in this study. A few DVAs are not detected on CT/MRI, but may be seen in the venous phase of angiography $[21,37]$. Most DVA and CM cases have a benign presentation and clinical process, and most of them are discovered accidentally. However, CM is occult in angiography and most researchers consider that MRI is sensitive to both DVAs and CMs. As a result, we only used the MRI data to study the association between DVA and concomitant CM. Finally, because cases were identified by searching the diagnosis reports in the computer image system of Beijing Tiantan Hospital, DVA and CM that were missed on initial diagnosis would have been excluded from the study. To test the extent of this problem, we reviewed an additional 100 randomly selected MRI examinations without DVA or CM diagnosis. None of these cases revealed DVA or CM findings, suggesting that the case selection method of this study was unlikely to have missed cases of DVA or CM. It is unlikely that our selection procedures significantly impacted the results.

\section{Conclusion}

As common cerebral vascular malformations, CMs are prone to coexist with DVAs. Concurrent CM is more likely when a DVA has three or more medullary veins that are visible simultaneously in at least one MRI section, when DVAs are infratentorial, and when multiple DVAs are present. Therefore physicians should be alerted that presence of DVA increases the likelihood of present or future CM formation. For all DVA cases, follow up MRI evaluation is warranted to monitor possible CM development.

\section{Competing interests}

The authors declare that they have no competing interests.

\section{Authors' contributions}

Dr. GM was responsible for the study design, statistical analysis of the data, and the drafting of this article. Dr. CB and Dr. TY were responsible for collection of the clinical and imaging data, and Dr. XL was responsible for the literature review. Dr. ZW was responsible for data interpretation, and review and revision of the draft. Dr. JTZ and Dr. JZZ were responsible for the study design, critical revision of the draft, and the final approval of the version to be published. All authors read and approved the final manuscript.

\section{Acknowledgements}

The study received financial support from the Beijing Nova program of science and technology. The funding organizations had no role in development of the study or the drafting of the manuscript for publication.

Received: 28 November 2013 Accepted: 11 March 2014

Published: 15 March 2014

\section{References}

1. Russell DS Rubinstein LJ: Pathology of Tumors of the Nervous System. London: Edward Arnold; 1951:78-79.

2. Koc K, Anik I, Akansel Q, Anik Y, Ceylan S: Massive intracerebral haemorrage due to developmental venous anomaly. Br J Neurosurg 2007, 21(4):403-405.

3. Cortes VJJ, Concepcion AL, Ballenilla MF, Gallego LJI, Gonzalez-Spinola SGJ: Cerebral cavernous malformations: spectrum of neuroradiological findings. Radiologia (Panama) 2012, 54(5):401-409. 
4. Topper R, Jurgens E, Reul J, Thron A: Clinical significance of intracranial developmental venous anomalies. J Neurol Neurosurg Psychiatry 1999, 67(2):234-238

5. Fushimi Y, Miki Y, Togashi K, Kikuta K, Hashimoto N, Fukuyama H: A developmental venous anomaly presenting atypical findings on susceptibility-weighted imaging. AJNR Am J Neuroradiol 2008, 29(7):E56.

6. Hon JM, Bhattacharya JJ, Counsell CE, Papanastassiou V, Ritchie V, Roberts RC, Sellar RJ, Warlow CP, Al-Shahi Salman R: The presentation and clinical course of intracranial developmental venous anomalies in adults: a systematic review and prospective, population-based study. Stroke 2009, 40(6):1980-1985.

7. Hong YJ, Chung TS, Suh SH, Park CH, Tomar G, Seo KD, Kim KS;Park IK: The angioarchitectural factors of the cerebral developmental venous anomaly; can they be the causes of concurrent sporadic cavernous malformation. Neuroradiology 2010, 52(10):883-891.

8. Ostertun B, Solymosi L: Magnetic resonance angiography of cerebral developmental venous anomalies: its role in differential diagnosis. Neuroradiology 1993, 35(2):97-104

9. Zabramski JM, Wascher TM, Spetzler RF, Johnson B, Golfinos J, Drayer BP, Brown B, Rigamonti D, Brown G: The natural history of familial cavernous malformations: results of an ongoing study. J Neurosurg 1994, 80(3):422-432.

10. McCormick WF, Hardman JM, Boulter TR: Vascular malformations ("angiomas") of the brain, with special reference to those occurring in the posterior fossa. J Neurosurg 1968, 28(3):241-251.

11. Dillon WP: Cryptic vascular malformations: controversies in terminology, diagnosis, pathophysiology, and treatment. AJNR Am J Neuroradiol 1997 18(10):1839-1846.

12. Petersen TA, Morrison LA, Schrader RM, Hart BL: Familial versus sporadic cavernous malformations: differences in developmental venous anomaly association and lesion phenotype. AJNR Am J Neuroradiol 2010, 31(2):377-382.

13. Plummer NW, Zawistowski JS, Marchuk DA: Genetics of cerebral cavernous malformations. Curr Neurol Neurosci Rep 2005, 5(5):391-396.

14. Porter RW, Detwiler PW, Spetzler RF, Lawton MT, Baskin JJ, Derksen PT, Zabramski JM: Cavernous malformations of the brainstem: experience with 100 patients. J Neurosurg 1999, 90(1):50-58.

15. Perrini $P$, Lanzino $G$ : The association of venous developmental anomalies and cavernous malformations: pathophysiological, diagnostic, and surgical considerations. Neurosurg Focus 2006, 21(1):e5.

16. Porter PJ, Willinsky RA, Harper W, Wallace MC: Cerebral cavernous malformations: natural history and prognosis after clinical deterioration with or without hemorrhage. J Neurosurg 1997, 87(2):190-197.

17. Abe T, Singer RJ, Marks MP, Norbash AM, Crowley RS, Steinberg GK: Coexistence of occult vascular malformations and developmental venous anomalies in the central nervous system: MR evaluation. AJNR Am J Neuroradiol 1998, 19(1):51-57.

18. Hegde AN, Mohan S, Lim CC: CNS cavernous haemangioma: "popcorn" in the brain and spinal cord. Clin Radiol 2012, 67(4):380-388.

19. Zhang P, Liu L, Cao Y, Wang S, Zhao J: Cerebellar cavernous malformations with and without associated developmental venous anomalies. BMC Neurol 2013, 13:134.

20. Maeder P, Gudinchet F, Meuli R, de Tribolet N: Development of a cavernous malformation of the brain. AJNR Am J Neuroradiol 1998, 19(6):1141-1143.

21. Hussain JZ, Ray A, Hughes DG, Leggate JR: Complex developmental venous anomaly of the brain. Acta Neurochir (Wien) 2002, 144(5):501-504

22. Wilson CB: Cryptic vascular malformations. Clin Neurosurg 1992, 38:49-84

23. Rothbart D, Awad IA, Lee J, Kim J, Harbaugh R, Criscuolo GR: Expression of angiogenic factors and structural proteins in central nervous system vascular malformations. Neurosurgery 1996, 38(5):915-924. discussion 924-5.

24. Sirin S, Kahraman S, Gocmen S, Erdogan E: A rare combination of a developmental venous anomaly with a varix. Case report. J Neurosurg Pediatr. 2008, 1(2):156-159.

25. Guclu B, Ozturk AK, Pricola KL, Seker A, Ozek M, Gunel M: Cerebral venous malformations have distinct genetic origin from cerebral cavernous malformations. Stroke 2005, 36(11):2479-2480.

26. Wilms G, Bleus E, Demaerel P, Marchal G, Plets C, Goffin J, Baert AL: Simultaneous occurrence of developmental venous anomalies and cavernous angiomas. AJNR Am J Neuroradiol 1994, 15(7):1247-1254. discussion 1255-7.

27. Mindea SA, Yang BP, Shenkar R, Bendok B, Batjer HH, Awad IA: Cerebral cavernous malformations: clinical insights from genetic studies. Neurosurg Focus 2006, 21(1):e1.
28. Cakirer S: De novo formation of a cavernous malformation of the brain in the presence of a developmental venous anomaly. Clin Radiol 2003, 58(3):251-256

29. Little JR, Awad IA, Jones SC, Ebrahim ZY: Vascular pressures and cortical blood flow in cavernous angioma of the brain. J Neurosurg 1990, 73(4):555-559.

30. Truwit CL: Venous angioma of the brain: history, significance, and imaging findings. AJR Am J Roentgenol 1992, 159(6):1299-1307.

31. Campeau NG, Lane Jl: De novo development of a lesion with the appearance of a cavernous malformation adjacent to an existing developmental venous anomaly. AJNR Am J Neuroradiol 2005, 26(1):156-159.

32. Omodaka S, Fujimura M, Endo T, Inoue T, Shimizu H, Tominaga T: De novo formation of orbital cavernous malformation 9 years after surgical management of dural arteriovenous fistula in the anterior middle fossa: case report. Neurol Med Chir (Tokyo) 2010, 50(4):324-327.

33. Clatterbuck RE, Elmaci I, Rigamonti D: The juxtaposition of a capillary telangiectasia, cavernous malformation, and developmental venous anomaly in the brainstem of a single patient: case report. Neurosurgery 2001, 49(5):1246-1250.

34. Labauge P, Laberge S, Brunereau L, Levy C, Tournier-Lasserve E: Hereditary cerebral cavernous angiomas: clinical and genetic features in 57 French families. Societe Francaise de Neurochirurgie. Lancet 1998 352(9144):1892-1897.

35. Gallione CJ, Pasyk KA, Boon LM, Lennon F, Johnson DW, Helmbold EA, Markel DS, Vikkula M, Mulliken JB, Warman ML: A gene for familial venous malformations maps to chromosome $9 p$ in a second large kindred. J Med Genet 1995, 32(3):197-199.

36. Campbell PG, Jabbour P, Yadla S, Awad IA: Emerging clinical imaging techniques for cerebral cavernous malformations: a systematic review. Neurosurg Focus 2010, 29(3):E6.

37. Rigamonti D, Spetzler RF, Medina M, Rigamonti K, Geckle DS, Pappas C: Cerebral venous malformations. J Neurosurg 1990, 73(4):560-564.

doi:10.1186/1471-2377-14-50

Cite this article as: Meng et al:: The association between cerebral developmental venous anomaly and concomitant cavernous malformation: an observational study using magnetic resonance imaging. BMC Neurology 2014 14:50

\section{Submit your next manuscript to BioMed Central and take full advantage of:}

- Convenient online submission

- Thorough peer review

- No space constraints or color figure charges

- Immediate publication on acceptance

- Inclusion in PubMed, CAS, Scopus and Google Scholar

- Research which is freely available for redistribution 